\title{
Philosophiques
}

\section{Origines et fondements philosophiques de la relativité : les conceptions de Mach, Galilée et Einstein}

\section{Jacques Montminy}

Volume 22, numéro 1, printemps 1995

URI : https://id.erudit.org/iderudit/027306ar

DOI : https://doi.org/10.7202/027306ar

Aller au sommaire du numéro

Éditeur(s)

Société de philosophie du Québec

ISSN

0316-2923 (imprimé)

1492-1391 (numérique)

Découvrir la revue

Citer cet article

Montminy, J. (1995). Origines et fondements philosophiques de la relativité : les conceptions de Mach, Galilée et Einstein. Philosophiques, 22(1), 21-34.

https://doi.org/10.7202/027306ar
Résumé de l'article

L'article qui suit se propose de montrer que le concept de « relativité » trouve, dans les conceptions de Mach, de Galilée et d'Einstein, une origine et un fondement philosophiques différents. En mettant ainsi en évidence les différences fondamentales qui séparent ces conceptions, il s'oppose en particulier à toute interprétation visant à faire de la théorie de la relativité d'Einstein, un « prolongement » de la philosophie positiviste de Mach et du principe galiléen de relativité. 


\title{
ORCGNESE FONDEMENS PHIIOSOPHIOUES DELARRATIIIIE: IES CONCPPIONS DE MACH, GAIIIFE ET ENSTEN
}

\author{
par \\ Jacques Montminy
}

\begin{abstract}
RÉSUMÉ : L'article qui suit se propose de montrer que le concept de « relativité » trouve, dans les conceptions de Mach, de Galilée et d'Einstein, une origine et un fondement philosophiques différents. En mettant ainsi en évidence les différences fondamentales qui séparent ces conceptions, il s'oppose en particulier à toute interprétation visant à faire de la théorie de la relativité d'Einstein, un « prolongement » de la philosophie positiviste de Mach et du principe galiléen de relativité.
\end{abstract}

ABSTRACT : The following paper analyses relativity's philosophical origin and fundament through the conceptions of Mach, Galileo and Einstein. It emphasizes the fundamental differences that lie between these conceptions thus opposing all interpretations suggesting that Einstein's theory of relativity can be properly described as being a mere « extension » of Mach's positivism and Galileo's principle of relativity.

Il est d'usage de souligner les liens qui unissent les idées d'Einstein à celles de Mach et de Galilée. Ainsi la théorie de la relativité est-elle le plus souvent présentée comme constituant une sorte de prolongement à la fois de la critique positiviste de Mach, à travers la définition opérationnelle du temps. l'abandon de l'éther et la relativisation de l'inertie, et du principe galiléen de relativité, par la double extension qu'elle donne à ce principe : la première, au domaine de l'optique, pour donner la relativité restreinte, et la deuxième au mouvement accéléré pour la relativité générale. Un tel usage toutefois est trompeur dans la mesure oủ il cache des différences fondamentales tant sur le plan philosophique que sur celui de la physique proprement dite. De fait, on peut se demander si les nombreux débats philosophiques soulevés par la théorie d'Einstein, dont, en particulier, ceux qui ont entouré la question de 
l'origine de la relativité restreinte ${ }^{\mathrm{I}}$ et celle du « statut » des effets relativistes ${ }^{2}$, ne sont pas en partie reliées à une perception insuffisante de ces différences. Le texte qui suit compare les conceptions des origines et des fondements philosophiques de la relativité de Mach, Galilée et Einstein. Il développe le

I. Cette controverse a été déclenchée par des prises de position d'Einstein de plus en plus éloignées du positivisme de Mach, et apparaissant incompatibles avec des déclarations antérieures, qui avaient accrédité une interprétation positiviste de la relativité restreinte où l'expérience de Michelson-Morley jouait un rôle tout à fait fondamental. De fait cette polémique a pris l'allure d'une véritable « affaire » où l'on a mis notamment en doute l'honnêteté d'un parent de Mach de même que la fiabilité des souvenirs d'Einstein quant à l'origine réelle de sa théorie. Enfın, elle a donné lieu à diverses autres thèses dont celle en particulier d'un revirement fondamental dans l'évolution de la pensée d'Einstein - la thèse des deux Einstein, celui « empiriste » de la relativité restreinte et celui « rationaliste » de la relativité générale, thèse qui a elle-même été contestée.

Concernantles positions épistémologiques d'Einstein on peut consulter les textes suivants, réunis dans A. Einstein, Ideas and Opinions, Crown Publishers, Wings Books, New Jersey, USA, 1954 : «Principles of Theoretical Physics 》, p. 220223; «Principles of Research », p. 224-227; «On the Method of Theoretical Physics », p. 220-276; «Physics and Reality » p. 290-323; « The Fundaments of Theoretical Physics », p. 323-335.

Concernant la relation entre Mach et Einstein et la question de l'origine de la relativité restreinte, on peut consulter notamment : $\mathrm{R}$. Itagaki, « Why did Mach reject Einstein's Theory of Relativity », paru dans Historia Scientiarum : International Journal of the History of Science Society of Japan $n^{\circ} 22$, I982, p. 81-95. M. Katsumori, «The Theories of Relativity and Einstein's Philosophical Turn », paru dans Studies in History and Philosophy of Science, déc. 1992, vol. 23, $\pi^{\circ} 4$, p. 557-592. P. Thuillier, D'Archiméde à Einstein, Paris, Fayard, collection « Le Temps des sciences », Ig88, p. 360-373. L. S. Feuer, Einstein et le conflit des générations, Paris, Presses universitaires de France, 1978, p. 80, $105^{-109}$ et 306-313. M. Paty, Einstein philosophe, Paris, Presses universitaires de France, collection « Philosophie d'aujourd'hui », I993, P. I10-130, $162-63,349-365$.

2. Cette deuxième controverse, quant à elle, a été soulevée par une prise de position très nette d'Einstein, en faveur du caractère « réel » de la dilatation du temps et de la contraction des longueurs, une prise de position jugée par plusieurs comme incompatible avec le concept même de relativité. Ce débat, qui s'est cristallisé autour du célèbre paradoxe des jumeaux a donné lieu à des thèses allant du faux paradoxe solutionné par la relativité générale - c'est l'interprétation « orthodoxe » à la contradiction pure et simple et au refus d'accorder un caractère absolu aux effets relativistes, en passant par une thèse intermédiaire affirmant le caractère « réel » de la dilatation du temps, en même temps que celui « apparent » de la contraction des longueurs.

On trouvera un exposé synthétique de ces thèses dans S. J. Prokhovnik, La Logique de la relativité restreinte, Paris, Gauthiers-Villars, 1969. 
point de vue selon lequel les liens qui relient ces conceptions relèvent, en un sens, moins d'une communauté de pensée que du hasard d'une rencontre, comme si leurs chemins s'étaient simplement croisés en un point : celui de la relativité du mouvement.

L'interprétation visant à faire de la relativité d'Einstein un « simple » prolongement de celles de Mach et de Galilée soulève divers problèmes, dont celui, notamment, du caractère « révolutionnaire » de la théorie d'Einstein. Elle soulève de plus une difficulté particulière due au fait qu'elle implique non pas « un » prolongement mais « deux », posant ainsi le problème de déterminer, tant quantitativement que qualitativement, l'apport exact des idées de Mach et de Galilée par rapport à celles d'Einstein.

Cette difficulté n'est d'ailleurs pas étrangère à la question de l'origine de la théorie de la relativité. On sait qu'Einstein, dans la première présentation qu'il a faite de sa theorie dans son mémoire de igo5 - il s'agit alors uniquement de la relativité restreinte - fonde celle-ci sur deux postulats, l'un étant le principe de relativité et l'autre celui de la constance de la vitesse de la lumière dans le vide $^{3}$. Or on retrouve précisément des interprétations contradictoires du rôle respectif de ces postulats. Ainsi retrouve-t-on une interprétation qui met l'accent sur le postulat de la relativité du mouvement, attribuant à celui de la constance du mouvement de la lumière la valeur d'un simple cas particulier ${ }^{4}$ tandis qu'une autre, à l'inverse, met l'accent sur le « fait » de la constance de la vitesse de la lumière; de ce fait, elle déduit, logiquement, la nécessité de modifier le principe galiléen, en remplaçant les transformations galiléennes par celles de Lorentz ${ }^{5}$. Enfin on retrouve aussi des interprétations qui accordent une égale importance aux deux postulats, mais qui laissent cependant entier le problème de la nature exacte de leur relation ${ }^{6}$. Si l'on ajoute à cela le fait que le premier de ces postulats relève de la relativité galiléenne, tandis que le

3. «On the Flectrodynamics of Moving Bodies », dans H.A. Lorentz et als. (dirs), The Principle of Relativity, New York, Dover Publications, 1952, p. 37-38.

4. Cette interprétation s'appuie sur un syllogisme du type suivant : les lois de la nature sont invariantes par rapport au mouvement uniforme, or la vitesse de la lumière est une loi de la nature, donc la vitesse de la lumière est invariante par rapport au mouvement uniforme. Einstein lui-même s'appuiera sur une telle interprétation, notamment dans La Relativité, Paris, Payot, collection « Petite bibliothèque Payot $\gg$ n 62, I98I, p. 20-28.

5. C'est l'interpretation que fera par exemple Max Born dans Einstein's Theory of Relativity, New York, Dover publications, 1962, p. 225-226.

6. Voir en particulier l'interprétation de T. M. Helliwell qui pose la difficulté supplémentaire de concevoir une combinaison des deux postulats qui sont « révolutionnaires » alors que chacun des deux pris séparément est posé comme etant tout à fait banal [innocent-soundingl dans An Introduction to Special Relativity, Boston, Allyn and Bacon, 1966, p. 20-21. 
deuxième relève, lui, d'une lecture positiviste de la relativité restreinte, on peut soupconner qu'il ne soit pas inutile de clarifier l'origine et les fondements des conceptions de Mach, Galilée et Einstein concernant la relativité. C’est ce que nous allons maintenant tenter de faire, en commencant par celle de Mach.

Le chemin qui a conduit Mach à la relativité part de la philosophie ou. plus précisément, de cette branche de la philosophie que constitue l'épistémologie: c'est un chemin qui a déjà été emprunté par des philosophes idéalistes tels que Leibniz et Berkeley et dont on peut retracer les origines jusque dans l' Antiquité, dans la célèbre affirmation du sophiste grec Protagoras, posant que « l'homme est la mesure de toute chose ». C'est en un sens le chemin le plus rapide des trois, celui qui mène le plus directement à la relativité du mouvement, dans la mesure où il se fonde sur le seul principe de la relativité, ou mieux, du caractère « relationnel » de toute connaissance humaine, l'idée, plus précisément, que l'on ne peut connaître les choses qu'à travers les relations que nous entretenons avec elles. Dans l'esprit de Mach ces relations constituent le « premier » fait. Aussi Mach reprochera-t-il à Newton d'avoir dérogé à son projet initial de ne considèrer que les faits en maintenant un temps et un espace indépendants, opposant aux absolus newtoniens l'interdépendance de toutes choses :

1... we reach our ideas of time in and through the interdependance of things on another?

[.] we must not forget that all things in the world are connected with one another and depend on one another, and that we ourselves and all our thoughts are also a part of nature.

C'est ce raisonnement appliqué au cas particulier du mouvement qui va conduire Mach jusqu'aux portes, pour ainsi dire, de la relativité générale d'Einstein. La simple règle méthodologique du recours aux faits et à l'observation entraîne déjà en effet l'impossibilité de déterminer, par l'observation directe, l'état de mouvement « réel » d'un corps seul, isolé dans un espace par ailleurs parfaitement vide. De façon plus générale, elle conduit à une modification de la conception spontanée du mouvement défini comme étant un changement de lieu, en établissant qu'une observation quelle qu'elle soit ne pourra jamais donner un tel changement à l'état «pur », une sorte de changement de lieu «en général », dans un espace absolu : un changement de lieu observé sera toujours un changement de lieu par rapport à un autre lieu, celui par exemple de l'observateur. Aussi le mouvement devra-t-il devenir pour la mécanique un changement de distance plutôt qu'un changement de lieu. En faisant ainsi l'objet d'une cinématique, le mouvement deviendra une pure

7. E. Mach, The Science of Mechanics, LaSalle (Illinois), The Open Court, I960, p. 274.

8. E. Mach, ibid, p. 273. 
relation, tout à fait réversible et indépendante en quelque sorte de la réalité même du mouvement : un objet ne pourra en effet modifier sa distance par rapport a un autre sans que celui-ci ne voit la sienne modifier de la même manière, et ce peu importe lequel des deux se trouve réllement en mouvement. Un objet ne sera alors en mouvement que par rapport à un autre. En ce sens-là la loi d'inertie peut déjà paraître relever de la métaphysique plus que de la physique dans la mesure où elle se prononce précisément sur l'état réel de mouvement $d^{x} u n$ corps seul dont elle prédit la caractère constant de la vitesse et celui rectiligne de la trajectoire.

Toutefois, l'importance attribuée par Mach à la notion de relation et d'interdépendance va lui permettre d'aller plus loin qu'une relativisation purement « optique » du mouvement. Une fois admis en effet le caractère relatif du mouvement, il devient alors nécessaire de poser que des objets possédant le mème état de mouvement seront en situation de repos les uns par rapport aux autres, de sorte qu'à l'intérieur d'un système en mouvement tout devrait se passer comme si le système était au repos. Cela Newton, comme avant lui Galilée, l'acceptait déjà, mais pour le seul cas du mouvement uniforme; cette relativité du mouvement ne tenait plus à ses yeux lorsqu'il était question d'un mouvement accéléré : dans un système en rotation, par exemple, les éléments sont bien en repos relatif et pourtant le mouvement se manifeste quand même, par l'apparition notamment de forces centrifuges. Ainsi Newton, en s'appuyant sur sa fameuse expérience d'un seau rempli d'eau et en rotation au bout d'une corde ${ }^{9}$ considérera-t-il avoir trouvé la limite de la relativisation du mouvement et un moyen en même temps de « voir » un mouvement réel et absolu.

À ce raisonnement, Mach rétorquera que pas plus le seau de Newton que la Terre elle-même ne constituent des systèmes isolés en rotation et que personne en fait n'a jamais pu observer un tel système, et encore moins y faire des expériences. En somme, pour Mach, l'hypothèse qui reste le plus près des faits, c'est celle qui reconnait précisément le caractère relatif de toute rotation. Aussi n'hésitera-t-il pas à affırmer que les effets reliès à la rotation de la Terre, tels par exemple l'aplatissement des pôles et la déviation du plan du pendule de Foucault, sont des effets de la rotation de la Terre relativement aux étoiles et non de la rotation de la Terre en elle-même, de sorte que ces mêmes effets devraient tout autant apparaître sur une Terre immobile, autour de laquelle

9. Voir à ce sujet A. Koyré, Etudes newtoniemnes, Paris, Gallimard-NRF, ig68, p. 123-I28, et 126 . 
tourneraient les étoiles, un raisonnement sur lequel Einstein s'appuiera pour élaborer sa théorie de la relativité générale ${ }^{\mathrm{IO}}$.

Si la relativité du mouvement apparaît chez Mach comme la conséquence logique d'un principe général d'ordre philosophique, elle apparaît rattachée chez Galilée à une thèse astronomique bien précise et bien particulière : celle de Copernic. La thèse copernicienne soulève en effet des problèmes considérables, dont celui de comprendre comment une Terre, lancée dans l'espace dans une course folle, peut présenter toutes les apparences d'une Terre immobile, comment un objet, par exemple, lancé à la verticale, peut retomber à l'endroit d'où il a été lancé, ou comment encore un objet peut franchir, par rapport à la Terre, la mème distance, qu'il soit lancé dans la même direction que le mouvement de la Terre ou dans la direction contraire. Copernic et d'autres, comme Giordano Bruno, sentent bien que la solution passe par une relativisation du mouvement et une analogie entre la situation des humains sur la Terre, et celle des passagers d'un navire voguant sur une eau calme ${ }^{\mathrm{II}}$.

Mais comment? Une simple comparaison en effet ne saurait tenir lieu de preuve : rien ne garantit que l'on puisse transposer le cas, somme toute bien particulier d'un bateau voguant sur l'eau, à l'échelle de la Terre, voire à celle de tout l'univers! Pour rendre la thèse de Copernic « rationnelle », encore faut-il pouvoir expliquer pourquoi et comment le mouvement peut être équivalent au repos et disparaître en quelque sorte " physiquement »!

Ce sera le grand mérite de Galilée que de fournir cette explication et de lui donner en plus une solide base expérimentale. Mais pour ce faire il devra modifier considérablement la conception du mouvement de ses contemporains, une conception issue de la philosophie d'Aristote. Il devra notamment supprimer l'opposition aristotélicienne entre mouvement naturel et violent et, surtout, introduire deux concepts révolutionnaires : celui d'inertie et celui de la superposition de mouvements. Ainsi le mouvement, qui était pour Aristote une action - celle de passer d'un lieu à un autre deviendra, pour Galilée, un état, quelque chose qui se conserve de lui-même « naturellement » sans qu'il soit nécessaire de faire intervenir un mécanisme particulier tel que celui imaginé par Aristote pour expliquer la persistance du mouvement horizontal, et qui supposait une sorte de « rétroaction » de l'air ambiant poussé par le mobile. De plus, les mouvements, qu'ils soient de même

Io. La nature exacte du lien reliant ce qu'Einstein lui-même nommera le «principe de Mach » et la relativité générale n'est cependant pas sans soulever quelques difficultès. Voir notamment J. Gribbin, $A$ la poursuite du big bang, Editions du Rocher, 1991, p. 123-138; M. Paty, op. cit., p. 210-220.

II. Lire à ce sujet A. Koyré, « Le Problème physique du copernicianisme » dans Etudes galileennes, Paris, Hermann, 1966, p. 165-186. 
direction, de direction contraire ou encore perpendiculaires les uns aux autres, vont pouvoir s'additionner et «fusionner » en un seul mouvement, à l'intérieur duquel ils pourront continuer d'exister de facon indépendante les uns des autres et être perçus comme tels.

C'est cette nouvelle mécanique que Galilée va opposer aux adversaires de la thèse copernicienne et c'est grâce à elle que l'équivalence du mouvement plus précisément du mouvement rectiligne uniforme - et du repos pourra devenir :

[...I une propriété de portée générale, connue aujourd'hui sous le nom de principe de relativité galiléen, et selon laquelle les lois de la mécanique sont les mèmes - et par conséquent les phénomènes mécaniques se déroulent de la même manière - relativement à deux référentiels dont l'un est animé d'une translation rectiligne uniforme par rapport à l'autre ${ }^{\mathrm{I2}}$.

On peut déjà ici clarifier une différence fondamentale entre la relativité de Mach et celle de Galilée. La relativité du mouvement n'est en effet dans la pensée de Mach rien de plus qu'un « exemple » de relativité. Elle constitue certes une démonstration saisissante de la viabilité d'une approche positiviste du type de celle de Mach, mais elle n'a sur le plan logique d'autre valeur que celle d'une application à un cas particulier - celui précisément du mouvement - d'un principe général imposant à toute affirmation scientifique, quelle qu'elle soit, de rester « dans » le réseau de relations à travers lequel ses objets lui sont donnés. En ce sens-là la conception de Mach constitue bien une «philosophie de la relativité » qui rejette toute vérité absolue et ne voit dans les théories scientifiques qu'un recoupement plus ou moins simple et commode des faits. Aussi la thèse copernicienne ne saurait-elle échapper à cette règle et être plus « vraie » que celle de Ptolémée à laquelle elle s'oppose :

Both views are, indeed, equally correct; only the latter is more simple and more practical ${ }^{\text {I3 }}$.

Or Galilée, précisément, était tout à fait convaincu, lui, de la vérité de la thèse copernicienne et il entendait bien prouver à ses contemporains que c'était Copernic et non Ptolèmée qui avait raison. Sa nouvelle mécanique constituait à ses yeux un argument de poids en faveur de la thèse héliocentrique, un argument qui s'ajoutait à ceux tirés des observations qu'il avait faites au télescope, en particulier celles des satellites de Jupiter et des phases de Vénus. Dans l'esprit de Mach le principe galiléen de relativité prouvait moins le mouvement de la Terre autour du soleil que l'impossibilité de prouver un tel mouvement, un point du vue, partagé par Poincaré, et qui conduit à une interprétation bien particulière du procès de Galilée:

I2. J. Gapaillard, Et pourtant elle toume, Paris, Seuil, 1993, p. I92.

I3. E.Mach, op. cit., p. 284. 
N'est-on pas allé jusqu'à reprocher à Poincaré de réhabiliter le système de Ptolémée et de justifier la condamnation de Galilée! Du moins semblait-il bien renvoyer dos à dos Galilée et ses juges ${ }^{\mathrm{I} 4}$.

Apparemment les juges, lors de ce procès se seraient bien accommodés d'une telle interprétation et alors il n'est pas impossible que le procès lui-même eût pu être évité. Mais, précisément, l'approche de Galilée ne relevait pas d'une approche positiviste et cela ses juges l'ont bien vi ${ }^{15}$ !

Le chemin qui conduira Einstein à sa théorie de la relativité ressemble, à certains égards, à celui de Galilêe. D'une part, la situation d'Einstein tentant d'établir la légitimité du système de Maxwell à travers l'indétectabilité du mouvement de la Terre - l'échec de l'expérience de Michelson-Morley - n'est pas sans rappeler celle de Galilée tentant de légitimer le système de Copernic! D'autre part, la relativité du mouvement ne joue pas non plus chez Einstein le rôle d'une simple illustration d'un principe genéral interdisant tout absolu, mais défınit bien au contraire, et ce, en dépit de l'expression même de « théorie de la relativité $\gg{ }^{\mathrm{I}}$, son propre absolu, à travers la constance de la vitesse de la lumière:

[...l la théorie de la relativité, si souvent mal comprise, non seulement n'a pas supprimé l'absolu, mais encore elle a fait ressortir mieux que jamais combien la physique est liée à un monde extérieur absolu [...] si l'espace et le temps perdent leur caractère d'absolu, ce dernier n'est pas le moins du monde expulsé de l'univers. Il s'est retiré plus loin, je veux dire dans la métrique du continu à quatre dimensions [...I par l'intermédiaire de la vitesse de la lumière ${ }^{17}$.

Encore une fois cependant la réalité est loin d'être aussi simple et l'on ne saurait s'en tenir à faire d'Einstein une sorte de deuxième Galilee. Mais, pour aller plus loin, il faut maintenant examiner pour lui-même le chemin qui a conduit Einstein à la relativité.

Au moment où Einstein élabore «sa s théorie de la relativité, bien des choses ont changé depuis l'époque de Galilee et de Newton:

14. J. Gapaillard, op. cit., P. 324. Voir nussi p. 11-14.

15. Voir O. Gingerich, «L'Affaire Galilée » dans Pour la science, n" 60, oct. I982, p. 69-79. Voir aussi J. Gapaillard, op.cit., p. $33^{8}$ : « la rotation de la terre avait, à ses yeux, tous les caractères d'un mouvement absolu, et c'est d'un tel mouvement qu'il soutenait lexistence 》.

16. On sait qu'Einstein a semble-t-il beaucoup hésitè à qualifier sa théorie de la relativité et considéré que le terme d'invariance serait plus juste que celui de relativité. Voir à ce sujet P.Thuilier, op. cit., p. 370-373; L. S. Feuer, op. cit., p. 105-109; M. Paty, Einstein philosophe, p. 182-183

17. M. Planck, Initiations a la physique, Paris, Flammarion, collection « Champs», Ig4I, p. 144 . 
Pendant la seconde moitié du $\mathrm{XIX}^{\mathrm{e}}$ siècle des idées nouvelles d'un caractère révolutionnaire ont été introduites en physique, qui ont fraye la voie à un nouveau point de vue philosophique, différent du point de vue mécanique ${ }^{18}$.

Les connaissances accumulées dans les domaines de l'électricité et du magnétisme ont introduit sur la scène de la physique de nouveaux acteurs : celui des ondes électromagnétiques, dont le mouvement de la lumière constitue le cas le plus familier, celui aussi de l'éther, qui doit servir de milieu de propagation pour ces ondes, et celui du champ qui fournit le modèle explicatif de base de cette propagation. Ces acteurs, dont Galilée lui-même ignorait complètement l'existence, sont de toute évidence appelés à jouer, en cette fin du XIX ${ }^{\mathrm{e}}$ siècle, un rôle fondamental. Toutefois la confrontation de la théorie avec les faits soulève peu à peu divers problèmes dont en particulier une difficulté tout à fait inattendue : diverses expériences, telles que celle de Fizeau et la fameuse expérience de Michelson-Morley, suggèrent en effet que la vitesse de la lumière pourrait constituer une constante universelle et garder toujours la même valeur quel que soit l'état de mouvement uniforme de l'observateur qui la mesure. Cela revient un peu, en somme, à affirmer l'existence d'un objet qui aurait la particularité de ne pas paraître plus petit lorsqu'on s'éloigne de lui, ou plus grand lorsqu'on s'en rapproche, mais qui garderait au contraire toujours la même taille apparente quelle que soit la distance à laquelle il se trouverait!

Certes, il y a bien des solutions possibles à ce paradoxe, comme d'imaginer un mécanisme caché qui puisse annuler l'effet attendu. Ce sera la voie que choisira Lorentz, invoquant une action de l'ether sur les objets ayant pour effet de contracter les objets dans la direction de leur mouvement et d'expliquer ainsi l'impossibilité de détecter un « vent » d'éther sur la Terre. D'autres, comme Poincaré, pointeront dans la direction du principe galiléen, $y$ voyant là la possibilité d'une explication plus générale et plus fondamentale ${ }^{\text {I9 }}$.

Mais la physique a elle-mème creusé un abîme entre la mécanique de Galilée et de Newton et l'électromagnétisme de Maxwell, entre le mouvement de la matière - celui des particules et des ondes « ordinaires » comme celles du son dans l'air ou des vagues sur l'eau - et celui de la lumière, entre la relativité nécessaire de la vitesse des corps et la constance nécessaire de la vitesse de la lumière. Et personne ne voit comment au juste franchir un tel abîme. Devant l'ampleur de la difficulté, certains physiciens n'hésitent pas à

I8. A. Einstein et L. Infeld, L'Evolution des idées en physique, Paris, Flammarion, collection «Champs », I983, p. II7.

19. Pour une comparaison détaillée des points de vue de Lorentz, Poincaré et Einstein, voir M. Paty, op. cit., p. 42-52, 82-83. 
suggérer de laisser tomber le principe galiléen tandis que d'autres jonglent avec l'idée de remettre en question la thèse copernicienne !

C'est à Einstein qu'il reviendra de denouer l'impasse. La solution, il la trouvera effectivement dans la direction pointée par Poincaré à savoir celle du principe galiléen, une direction qu'Einstein lui-même avait semble-t-il pressenti dès l'âge de 16 ans ! Mais pour lier la mécanique à l'optique, Einstein devra modifier la relativite galiléenne pour la rendre compatible avec le caractère particulier du mouvement de la lumière et des ondes électromagnétiques en général.

La vitesse de la lumière ne constitue pas en effet dans la théorie d'Einstein, une vitesse comme les autres, mais « la » vitesse de la Nature en quelque sorte, dans la mesure où elle constitue une vitesse maximale dans la Nature. De plus, la constance de la vitesse de la lumière est assurée physiquement par le fait que la propagation des ondes électromagnétiques est à la fois indépendante du mouvement de sa source et indépendante de tout milieu matériel de propagation, deux propriétés quejusque là la physique avait toujours séparées, l'une - l'indépendance quant à la source - étant réservee aux corps et l'autrel'indépendance quant au milieu de propagation - aux ondes.

Or ce caractère tout à fait particulier de l'interaction électromagnétique va exiger une modification importante de la relativité galiléenne. Le principe galiléen constitue en effet, un cas particulier d'invariance où tous les éléments d'un système sont modifiés de façon identique, de telle sorte que les relations entre ces élements demeurent inchangées: dans un système en mouvement tous les éléments participent au mouvement du système de telle sorte que l'on se retrouve dans une situation de repos relatif à l'intérieur du système. C'est un peu si l'on veut la même invariance que celle entre les quantités: $1 / 2,2 / 4,3 / 6,4 /$ 8 , etc.

Mais précisément dans un système en mouvement le mouvement de la lumière ne participe pas au mouvement du système et sa vitesse demeure inchangée non seulement à l'intérieur du système mais aussi à l'extérieur. La distinction même entre un point de vue intérieur et un point de vue extérieur, qui conditionne les mesures des vitesses des corps dans la relativité galiléenne, n'a plus cours dans le cas du mouvement de la lumière : un faisceau lumineux émis de l'intérieur d'un train en mouvement, ne verra pas la vitesse du train additionnée à la sienne, s'il est émis dans le sens de la direction du train, pas plus qu'il ne verra sa vitesse diminuée s'il est émis vers l'arrière du trair. Le faisceau, émis de l'intérieur du train, se comportera en fait exactement de la même façon que s'il pénétrait à l'intérieur du train après avoir été émis de l'exterieur. En d'autres termes, comme le mouvement de la lumière ne participe pas au mouvement du système auquel il est « lié », il se comporte par rapport à ce système comme un phénomène d'inertie. 
Cette différence fondamentale entre le mouvement de la lumière et celui de la matière nécessite une modification profonde du principe galiléen de relativité, que l'on formule souvent en disant qu'un observateur dans un système en mouvement uniforme ne peut pas détecter le mouvement de son système tant qu'il reste «à l'intérieur ». Car si la théorie d'Einstein prédit elle aussi l'indétectabilité du mouvement « de l'intérieur », c'est cependant pour une raison différente et selon un sens différent de celui du principe galiléen puisque la notion d'intérieur ne s'applique pas « directement » à la relativité d'Einstein, laquelle doit tenir compte du caractère absolu de la vitesse de la lumière dans un tel système. Ainsi un objet lancé verticalement dans un train en mouvement sera perçu de l'intérieur du train comme ayant une trajectoire verticale tandis qu'un observateur extérieur percevra, lui, une trajectoire parabolique. Mais un faisceau lumineux émis dans les mêmes conditions n'aura pas une trajectoire verticale pour l'observateur intérieur pas plus que pour l'observateur extérieur puisque son mouvement ne sera pas modifié par celui du train. La trajectoire sera donc celle d'un faisceau entrant de l'extérieur du train, par « en-dessous » et dans une direction verticale: une trajectoire oblique, dirigée dans la direction contraire à celle du mouvement du train et d'autant plus près d'une ligne horizontale que la vitesse du train sera plus grande. Comment alors« sauver » le principe de relativité et faire en sorte que tout, à l'intérieur du train, puisse encore se passer comme si le train était au repos? En se rendant compte que l'observateur à l'intérieur du train n'aura pas la même «définition » de la verticale que l'observateur extérieur et que, à l'intérieur du train, un plan vertical est un plan oblique - il serait parabolique dans le cas d'un mouvement accéléré - orienté dans la direction du mouvement du train. L'observateur dans le train jugera que le faisceau a été émis verticalement lorsque I'observateur extérieur jugera que le même faisceau a été émis selon une trajectoire oblique, et l'écart avec la verticale sera d'autant plus prononcé que la vitesse du train sera plus grande.

En d'autres termes, si, dans la théorie d'Einstein, un système en mouvement est équivalent à un système au repos ce n'est pas, comme dans la relativité galiléenne, parce que tous les éléments du système ont été modifiés de façon identique, mais parce que le système lui-même, son espace et son temps ont changé, comme si le « train » avait été modifié « physiquement», cormme s'il avait été tordu, étiré, comprimé, désynchronisé ${ }^{20}$.

C'est cette différence entre la relativité d'Einstein et celle de Galilée qui explique le fait que si Einstein, tout comme Galilée, maintient un absolu, celui

20. On peut mesurer ici l'insuffisance de la présentation qui est faite de la dilatation du temps, dans les ouvrages de vulgarisation sur la relativité restreinte, où l'on «montre » la trajectoire oblique d'un rayon lumineux émis verticalement et réfléchi à l'intérieur d'un système en mouvement uniforme, en s'appuyant sans 
de l'espace-temps, cet absolu toutefois est nettement plus abstrait et éloigné de notre univers quotidien - et cela sera encore plus vrai pour la relativité générale que pour la relativité restreinte - que ne l'était celui de Galilée. En ce sens-là Einstein a considérablement « aggravé » le problème dejà posé par la relativité du mouvement dans la physique classique. Déjà, la relativité galiléenne entraînait comme conséquence le fait que des observateurs dans des systèmes en mouvement relatif vivaient en quelque sorte dans des univers différents, où les vitesses et les trajectoires dépendaient entièrement du système à partir duquel on effectuait les mesures : dans les faits, cela revenait à affirmer que le mouvement créait littéralement des univers différents et qu'il y avait autant d'univers que d'états de mouvements! La solution d'Einstein, elle, creuse un écart encore plus grand entre ces univers, en leur attribuant des temps différents, une diffërence qui entraîne à son tour que dans ces univers, l'espace, le poids, la masse des objets varient eux aussi en fonction de la vitesse du système auquel ils sont liés. Aussi l'univers se transforme-t-il en une pluralité d'univers différents, des univers pourtant tous aussi réels les uns que les autres pour ceux qui y vivent!

Mais l'unité de la physique n'est pas perdue pour autant. De fait, le principe galiléen apportait déjà, en même temps que la mauvaise nouvelle de la relativité des vitesses et des trajectoires, la bonne nouvelle que la physique pouvait continuer d'exister et qu'elle pouvait même en un sens faire comme si le mouvement n'existait pas. Puisque tout sur la Terre se passait de la même façon, que la Terre soit en repos ou en mouvement, la physique était donc malgré tout possible : il restait de la place pour des lois universelles, indépendantes non seulement du temps et de l'espace, mais aussi du mouvement lui-même.

Cette interprétation, déjà présente dans la physique classique de Galilée et de Newton, va prendre dans la réflexion d'Einstein, qui, lui, a vu le principe galiléen échapper à la « tempête » de l'électromagnétisme, des dimensions tout à fait inattendues. De fait, le principe galiléen va acquérir aux yeux d'Einstein un tout nouveau statut. Conçu à l'origine pour expliquer une particularité du mouvement des corps et établir la vérité du système copernicien, ce principe va devenir une sorte de « méta-principe », une loi régissant les lois de la physique

autre explication sur le principe de relativité pour expliquer pourquoi et comment cette trajectoire peut devenir verticale pour l'observateur intérieur. Si l'on applique en fait tel quel le principe galiléen, la trajectoire du rayon observée à la fois par l'observateur extérieur et intérieur serait oblique, et de plus elle serait et ce, encore pour les deux observateurs, orientée non pas dans la direction du mouvement du train, mais dans la direction contraire! La situation « réelle » ne peut être illustrée qu'en remplaçant le système de coordonnées cartésiennes par un système oblique qui montre la « déformation » subie par le train à la suite de son mouvement. 
elles-mêmes, comme le faisaient dejjà d'autres principes comme celui de la conservation de l'énergie et le deuxième principe de la thermodynamique ${ }^{2 I}$.

C'est ce principe qui allait conduire Einstein au-delà de la relativité restreinte et l'orienter dans une recherche visant à déterminer un absolu qui serait tel qu'il échapperait non seulement au mouvement uniforme, mais à tout mouvement quel qu'il soit, et qui aboutirait à la théorie de la relativité générale.

D'une certaine façon c'est ici de Mach plus que de Galilée qu'Einstein se rapproche. Certes pas sur la question de l'absolu comme tel, mais sur le fait que le principe de relativité est associé tant dans la pensée de Mach que dans celle d'Einstein à une véritable « conception du monde », tandis qu'il reste, chez Galilée, attaché à une conception du mouvement, d'abord et avant tout. Einstein et Mach, en ce sens-là, sont plus philosophes que Galilée et c'est en tant que philosophes qu'ils se rejoignent. même si leurs philosophies s'opposent. Si la relativité a constitué le contenu fondamental de la philosophie de Mach, elle a constitué pour Einstein, le moyen pour arriver à une nouvelle conception de la réalité, une réalité dont il ne doutait pas qu'elle fût « une » et dont il cherchait l'unité dans le concept du champ :

On pourrait, de cette façon, creeer un arrière-plan philosophique nouveau. Son but final serait l'explication de tous les événements de la nature par des lois de structure toujours et partout valables [...] le champ étant la seule réalité... ${ }^{22}$.

Si les théories scientifiques ne sont pas à l'abri des débats philosophiques. c'est précisément parce qu'il n'y a pas de faits «bruts », indépendants de toute interprétation. La relativité du mouvement elle-même n'échappe pas à cette règle et ne constitue pas elle non plus une donnée « absolue ». C'est ce que l'on oublie trop facilement et qui explique le sentiment de gêne qu'il n'est pas rare de retrouver, dans les ouvrages de vulgarisation sur la relativité, lorsque vient le moment d'aborder la conception du temps et de l'espace newtoniens, comme si l'auteur sentait le besoin d'excuser Newton de ne pas avoir « vu » le caractère relatif de toute perception ! Mais pour ceux qui choisissaient Ptolémée à la place de Copernic, la relativité de Galilée ne s'imposait nullement : pire, elle était inutile. De même la relativité d'Einstein ne devenait-elle « évidente » qu'à partir du moment où l'on acceptait de faire les choix d'Einstein, dont celui de l'unité de la physique, tandis que celle de Mach. elle, découlait de son postulat « relationniste ». Aussi, si Mach, Galilée, et Einstein, ont tous les trois affirmé le caractère relatif du mouvement, c'est cependant à partir de chemins bien

2I. Voir à ce sujet « What is the Theory of Relativity » dans A. Einstein, op. cit., p. 227232, p. 228. Voir aussi M. Paty, op. cit., P. 421-23.

22. A. Einstein et L. Infeld, op. cit., p. 229-230. 
différents, et qui ne se sont peut-être finalement croisés que sur la question du mouvement.

Département de philosophie Cegep de Limoilou 